\title{
Thermal Hawking radiation of black hole with supertranslation field
}

\author{
Mikhail Z. Iofa \\ Skobeltsyn Institute of Nuclear Physics, \\ Moscow State University, Moscow 119992, Russia \\ E-mail: iofa@theory.sinp.msu.ru
}

ABSTRACT: Using the analytical solution for the Schwarzschild metric containing supertranslation field, we consider two main ingredients of calculation of the thermal Hawking black hole radiation: solution for eigenmodes of the d'Alambertian and solution of the geodesic equations for null geodesics. For calculation of Hawking radiation it is essential to determine the behavior of both the eigenmodes and geodesics in the vicinity of horizon. The equation for the eigenmodes is solved, first, perturbatively in the ratio $O(C) / M$ of the supertranslation field to the mass of black hole, and, next, non-perturbatively in the near- horizon region. It is shown that in any order of perturbation theory solution for the eigenmodes in the metric containing supertranslation field differs from solution in the pure Schwarzschild metric by terms of order $L^{1 / 2}=(1-2 M / r)^{1 / 2}$. In the non-perturbative approach, solution for the eigenmodes differs from solution in the Schwarzschild metric by terms of order $L^{1 / 2}$ which vanish on horizon. Using the simplified form of geodesic equations in vicinity of horizon, it is shown that in vicinity of horizon the null geodesics have the same behavior as in the Schwarzschild metric. As a result, the density matrices of thermal radiation in both cases are the same.

KeYwords: Black Holes, Models of Quantum Gravity

ARXIV EPRINT: 1708.09169 


\section{Contents}

1 Introduction 1

2 Generalization of the Schwarzschild metric containing the supertranslation field

\section{Introduction}

Recently there was a renewed interest in asymptotic symmetries at the null infinity, the BMS symmetries [1, 2]. The group of symmetries of asymptotically flat gravity, the BMS group, extends the Poincare group and is the semi-direct product of the Lorentz group and the normal abelian subgroup of supertranslations which generalize translations. The $B M S$ group can be enlarged to a group which also contains superrotations, singular supertranslations and local conformal transformations [3-5].

When acting on an asymptotically flat physical state the $B M S$ group of diffeomorphisms transforms it to another physical state preserving the asymptotic flatness. The BMS group contains coordinate transformations which are pure gauge transformations and also diffeomorphisms that change supertranslation field in the metric and provide the mapping of a physical state to another physical state $[6,7]$.

In paper [6], was obtained an important physical result that provided a metric decreases fast enough at infinity, in some neighborhood of Minkowski vacuum the $S$ matrix is invariant under an infinite-dimensional subgroup of $B M S^{+} \times B M S^{-}$, where $B M S^{ \pm}$are the groups acting at the future and past null infinities. The correlated result is that in the low energy gravitational scattering, because of the supertranslation invariance of the S-matrix, the local energy is conserved at any angle. In papers $[6,8]$ connection of an (in principle) observable gravitational memory effect with the change of vacua under the action of the BMS transformations was elucidated and connection with the soft graviton theorem [9] was established. Possible relation of the extended BMS group to the black hole information problem was discussed in [10].

In papers [7, 11] a method to construct a metric containing a finite supertranslation field in the bulk was developed. The metric was obtained by exponentiation of infinitesimal supertranslation diffeomorphism. When applied to the Schwarzschild metric, solution generating technic of $[7,11]$ provides generalization of the Schwarzschild metric containing supertranslation field $C(\theta, \varphi)$. 
Using the analytical solution of paper [7] we consider calculation of "hard" thermal Hawking radiation [12] of the Schwarzschild black hole with supertranslation field. In the present paper we consider the supertranslation field depending only on $\theta: C=C(\theta)$. Calculations are performed perturbatively in the ratio $O(C) / M$, where $M$ is the mass of black hole.

We follow the standard procedure of calculation of the Hawking radiation from a collapsing body [13-15]. We consider a massless scalar field propagating in the background of the Schwarzschild metric with a supertranslation field. Before the collapse begins, there is no radiation, and the system is in $i n$-vacuum with the $i n$-modes which have positive frequency at the past null infinity $I_{-}$. Quantization proceeds by expanding the field in the eigenmodes of the d'Alambertian written in the Schwarzschild metric with a supertranslation field. After the collapse begins, emission begins and is dominated by the modes which have left $I_{-}$with high frequency and propagate through the collapsing body just before the horizon was formed. The particle content of outgoing modes with respect to the modes at $I_{-}$is obtained by expanding the outgoing modes with respect io the basis of incoming modes. To determine the overlap between the outgoing and the ingoing modes, it is necessary to determine behavior of the outgoing modes near the horizon. We study solutions for the eigenmodes of the d'Alambertian with the Schwarzschild metric with supertranslation field in the limit $r \rightarrow 2 M$. We find that in any order in perturbation theory in $O(C) / M$, in the vicinity of horizon, solutions for the eigenmodes in the metric with supertranslation field differ from solutions in the pure Schwarzschild background by the terms of order $L^{1 / 2} O\left(C^{n} / M^{n}\right)$ where $L=(1-2 M / r)$. Next, we show that the same result is valid for the non-perturbative solution, in which case the difference between solutions for the eigenmodes in the pure Schwarzschild background, $\psi_{0}$, and in the Schwarzschild background with supertranslation field is $L^{1 / 2} \varphi(\theta, r) \psi_{0}$, where $L^{1 / 2} \partial_{r} \varphi$ is finite in the limit $L=0$. To calculate the overlap between the ingoing and outgoing modes, the wave packets representing particles are propagated backwards to $I_{-}$. Massless particles propagate following the null geodesics. We study the form of the null geodesics in vicinity of horizon. Despite a more complicated form because of $\theta$-dependence of the metric, in the near-horizon region the radial null geodesics differ from those in the pure Schwarzschild background by terms of order $(1-2 M / r)^{1 / 2}$.

Collecting these results we find that the Bogolubov coefficients giving the particle content of the out-modes through the in-modes and the density matrix of thermal radiation in the metric with supertranslation field are the same as in the pure Schwarzschild background.

\section{Generalization of the Schwarzschild metric containing the supertrans- lation field}

The generic final state of collapse of matter is a stationary space-time. In [7] was constructed a metric generalizing the Schwarzschild metric and containing supertranslation field. In the isotropic spherical coordinates in which the original Schwarzschild metric is 
written as

$$
d s^{2}=-\frac{(1-M / 2 \rho)^{2}}{(1+M / 2 \rho)^{2}} d t^{2}+(1+M / 2 \rho)^{4}\left(d \rho^{2}+\rho^{2} d \Omega^{2}\right),
$$

the metric containing the supertranslation field $C(\theta, \varphi)$ was obtained in a form

$$
\begin{aligned}
d s^{2}= & -\frac{\left(1-M / 2 \rho_{s}\right)^{2}}{\left(1+M / 2 \rho_{s}\right)^{2}} d t^{2} \\
& +\left(1+M / 2 \rho_{s}\right)^{4}\left(d \rho^{2}+\left(\left((\rho-E)^{2}+U\right) \gamma_{A B}+(\rho-E) C_{A B}\right) d z^{A} d z^{B}\right) .
\end{aligned}
$$

Here

$$
\rho_{s}(\rho, C)=\sqrt{\left(\rho-C-C_{00}\right)^{2}+D_{A} C D^{A} C}, .
$$

$C_{00}$ is the lowest constant spherical harmonic of $C(\theta, \varphi)$. In the following we do not write $C_{00}$ explicitly understanding $C \rightarrow C-C_{00}$. Covariant derivatives $D_{A}$ are defined with respect to the metric on the sphere $d s^{2}=d \theta^{2}+\sin ^{2} \theta d \varphi^{2}$. For technical simplicity we consider the case of $C$ depending only on $\theta, C=C(\theta)$.

The tensor $C_{A B}$ and the functions $U$ and $E$ are defined as

$$
\begin{aligned}
C_{A B} & =-\left(2 D_{A} D_{B}-\gamma_{A B} D^{2}\right) C, \\
U & =\frac{1}{8} C_{A B} C^{A B} \\
E & =\frac{1}{2} D^{2} C+C .
\end{aligned}
$$

The function $C$ has dimension of mass.

The non-zero Christoffel symbols are $\Gamma_{\varphi \theta}^{\varphi}=\cos \theta / \sin \theta, \Gamma_{\varphi \varphi}^{\theta}=-\cos \theta \sin \theta$. For $C_{A B}$ we obtain

$$
\begin{aligned}
C_{\theta \theta} & =-\left(C^{\prime \prime}-C^{\prime} \cot \theta\right), \\
C_{\varphi \varphi} & =\sin ^{2} \theta\left(C^{\prime \prime}-C^{\prime} \cot \theta\right), \\
C_{\varphi \theta} & =0 .
\end{aligned}
$$

Here prime denotes the derivative $\partial_{\theta}$. From (2.5)-(2.6) it follows that $C_{\varphi \varphi}=-\sin ^{2} \theta C_{\theta \theta}$. The functions $E(\theta)$ and $U$ are

$$
\begin{aligned}
& E=\frac{1}{2}\left(C^{\prime \prime}+C^{\prime} \cot \theta\right)+C, \\
& U=\frac{1}{4}\left(C^{\prime \prime}-C^{\prime} \cot \theta\right)^{2} .
\end{aligned}
$$

Equation $\rho_{s}\left(\rho_{H}, C\right)=M / 2$ defines location of the horizon $\rho_{H}$. For horizon to exist, condition $M^{2} / 4-D_{A} C D^{A} C>0$ should be fulfilled. In the case $C=C(\theta)$ condition of existence of horizon takes the form $M / 2>\left|C^{\prime}(\theta)\right|$.

Let us transform the metric (2.2) to the Schwarzschild variables introducing

$$
r=\rho_{s}(\rho, C)\left(1+\frac{M}{2 \rho_{s}(\rho, C)}\right)^{2} .
$$


Inverting this equation, we obtain

$$
\rho_{s}(\rho, C)=\frac{1}{2}(r-M+\sqrt{r(r-2 M)})
$$

In variable $r$ horizon is located at the point $r=2 M$.

Let us introduce notations

$$
\begin{aligned}
& L=1-\frac{2 M}{r}, \\
& K=r-M+r L^{1 / 2}, \quad \frac{d K}{d r}=\frac{K}{r L^{1 / 2}} .
\end{aligned}
$$

Using the relation (2.9), we have

$$
\frac{\left(1-M / 2 \rho_{s}\right)^{2}}{\left(1+M / 2 \rho_{s}\right)^{2}}=L, \quad\left(1+M / 2 \rho_{s}\right)^{4}=\frac{4 r^{2}}{K^{2}} .
$$

From the equation (2.9) written as

$$
\sqrt{(\rho-C)^{2}+D_{A} C D^{A} C}=\frac{K}{2},
$$

we obtain the expression for $\rho$ as a function of $r$ and $C$

$$
\rho=C+\frac{K}{2}\left(1-\frac{4(D C)^{2}}{K^{2}}\right)^{1 / 2} .
$$

Let us denote

$$
b=\frac{2 C^{\prime}}{K}
$$

so that (2.13) becomes

$$
\rho=C+\frac{K}{2} \sqrt{1-b^{2}}
$$

Because $K$ is the increasing function of $r, b$ has its maximum at $r=2 M$ equal to $2\left|C^{\prime}\right| / M$. Relation (2.15) is meaningful for $\left|C^{\prime}\right|<M / 2$.

Differentiating (2.13), we obtain

$$
d \rho=\frac{K}{2}\left[\left(b-\frac{b b^{\prime}}{\sqrt{1-b^{2}}}\right) d \theta+\frac{d r}{r L^{1 / 2} \sqrt{1-b^{2}}}\right] .
$$

Using that $U=C_{\theta \theta}^{2} / 4$, we transform the metric components $g_{\theta \theta}$ and $g_{\varphi \varphi}$ to a form

$$
\begin{aligned}
g_{\theta \theta} & =\left((\rho-E)^{2}+U\right) \gamma_{\theta \theta}+(\rho-E) C_{\theta \theta}=\left(\rho-E+\frac{C_{\theta \theta}}{2}\right)^{2} \\
& =\frac{K^{2}}{4}\left(\sqrt{1-b^{2}}-b^{\prime}\right)^{2}, \\
g_{\varphi \varphi} & =\left((\rho-E)^{2}+U\right) \gamma_{\varphi \varphi}+(\rho-E) C_{\varphi \varphi}=\left(\rho-E-\frac{C_{\theta \theta}}{2}\right)^{2} \sin ^{2} \theta \\
& =\frac{K^{2}}{4} \sin ^{2} \theta\left(b \cot \theta-\sqrt{1-b^{2}}\right)^{2} .
\end{aligned}
$$


Determinant of the angular part of the metric $(2.2), g_{\theta \theta} g_{\varphi \varphi}$, vanishes on the surfaces $\rho_{\mathrm{SH}}[7]$

$$
\left(\left(\rho_{\mathrm{SH}}-E\right)^{2}+U\right) \gamma_{\theta \theta} \pm\left(\rho_{\mathrm{SH}}-E\right) C_{\theta \theta}=0
$$

or, in variable $r$, on the surfaces $\sqrt{1-b^{2}}-b^{\prime}=0$ and $b \cos \theta-\sqrt{1-b^{2}} \sin \theta=0$. On these surfaces the inverse metric is undefined. Sufficient conditions on $C(\theta)$ ensuring that the matrix component $g_{\theta \theta}$ is non-zero for $r>2 M$ is

$$
\frac{M}{2}>\left(C^{2}+C^{\prime 2}\right)^{1 / 2}
$$

The component of the metric $g_{\varphi \varphi}$ is positive, if

$$
\frac{M}{2} \sin \theta>\left|C^{\prime}\right|
$$

These conditions, in particular, are fulfilled for $C(\theta)=\sum a_{n} P_{n}(\cos \theta)$, where $P_{n}(x)$ are Legendre polynomials, with a suitable choice of the coefficients $a_{n}$. For example, taking for $C(\theta)=a_{n} P_{n}(\cos \theta)$ we have $C^{\prime}(\theta)=a_{n} n\left(P_{n}(\cos \theta)-P_{n-1}(\cos \theta)\right) / \sin \theta$. At $\theta \sim 1$ this expression is bounded by $a_{n} n \times$ const, at $\theta \ll 1$ we have $C^{\prime} \simeq a_{n} \theta(n+1) / 2$, and both sufficient conditions on the components of the metric can be fulfilled.

The class of models with a supertranslation fields $C(\theta)$ contains the Kerr solution in which case $C_{\theta \theta}(\theta)=a / \sin \theta[18,19]$ and $C(\theta)=a_{2}+a_{1} \cos \theta+a \sin \theta$ with $a_{1}, a_{2}$ arbitrary.

Using the expressions (2.10), (2.12) and substituting the components of the metric $(2.17),(2.18)$ in $(2.2)$, we obtain the metric in Schwarzschild variables

$$
\begin{aligned}
d s^{2}= & -L d t^{2}+\frac{d r^{2}}{L\left(1-b^{2}\right)}+2 d r d \theta \frac{b\left(\sqrt{1-b^{2}}-b^{\prime}\right) r}{\left(1-b^{2}\right) L^{1 / 2}} \\
& +d \theta^{2} r^{2} \frac{\left(\sqrt{1-b^{2}}-b^{\prime}\right)^{2}}{1-b^{2}}+d \varphi^{2} r^{2} \sin ^{2} \theta\left(b \cot \theta-\sqrt{1-b^{2}}\right)^{2}
\end{aligned}
$$

Determinant of the metric (2.19) is

$$
|g|=r^{4} \sin ^{2} \theta \frac{\left(\sqrt{1-b^{2}}-b^{\prime}\right)^{2}}{1-b^{2}}\left(\sqrt{1-b^{2}}-b \cot \theta\right)^{2} .
$$

The inverse metric is

$$
\left(\begin{array}{cccc}
-L^{-1} & 0 & 0 & 0 \\
0 & L & -L^{1 / 2} b\left[r\left(\sqrt{1-b^{2}}-b^{\prime}\right)\right]^{-1} & 0 \\
0 & -L^{1 / 2} b\left[r\left(\sqrt{1-b^{2}}-b^{\prime}\right)\right]^{-1} & {\left[r\left(\sqrt{1-b^{2}}-b^{\prime}\right)\right]^{-2}} & 0 \\
0 & 0 & 0 & {\left[r^{2} \sin ^{2} \theta\left(\sqrt{1-b^{2}}-b \cot \theta\right)^{2}\right]^{-1}}
\end{array}\right)
$$


We introduce the following notations which we use below

$$
\begin{aligned}
\sqrt{|g|} & =\sqrt{g_{0}|\hat{g}|}=r^{2} \sin \theta \sqrt{|\hat{g}|} \\
\sqrt{|\hat{g}|} & =1+g_{1}+g_{2}+\cdots \\
& =1-\left(b^{\prime}+b \cot \theta\right)-\left(\frac{b^{2}}{2}-b^{\prime} b \cot \theta\right)+\cdots \\
& =1-\frac{D^{2} C}{K}+\frac{\left(D^{2} C\right)^{2}-C_{\theta \theta}^{2}-2(D C)^{2}}{K^{2}}+\cdots \\
g_{r r} & =\frac{1}{L} \hat{g}_{r r}, \quad g_{r \theta}=\frac{L^{1 / 2}}{r} \hat{g}_{r \theta}, \quad g^{r \theta}=-\frac{r}{L^{1 / 2}} \hat{g}^{r \theta}, \quad g_{\theta \theta}=r^{2} \hat{g}_{\theta \theta}, \\
\hat{g}^{r \theta} & =g_{1}^{r \theta}+g_{2}^{r \theta}+\cdots=b+b b^{\prime}+\cdots=\frac{2 C^{\prime}}{K}+\frac{2(D C)^{2}}{K^{2}}+\cdots, \\
f_{1} & =\sqrt{1-b^{2}}-b^{\prime}, \\
f_{2} & =\sin ^{2} \theta\left(\sqrt{1-b^{2}}-b \cot \theta\right) .
\end{aligned}
$$

\section{Equation for eigenmodes}

In this section we study solutions for the eigenmodes of the equation $\square(g) \psi=0$. Before the collapse begins, there is Minkowski vacuum at the past null infinity. A part of the modes propagete from the past null infinity through the collapsing body and go away to the future null infinity. The main contribution to the Hawking radiation is formed by modes that come through the collapsing body just before the horizon is formed in the vicinity of horizon $[13,15,16]$. We study behavior of the modes in the vicinity of the horizon $r=2 M$ both perturbatively and non-perturbatively in small parameter $O(C) / K \sim O(C) / M$ and prove that corrections to solutions in the pure Schwarzschild metric as functions of $r$ vanish at the horizon as $L^{1 / 2}=(1-2 M / r)^{1 / 2}$.

The operator $\square(g)$ is

$$
\begin{aligned}
\square= & \frac{1}{\sqrt{-g}} \partial_{\mu} \sqrt{|g|} g^{\mu \nu} \partial_{\nu} \\
= & g^{t t} \partial_{t}^{2}+\frac{1}{\sqrt{-g}} \partial_{r} \sqrt{-g} g^{r r} \partial_{r}+\frac{1}{\sqrt{-g}} \partial_{r} \sqrt{-g} g^{r \theta} \partial_{\theta}+\frac{1}{\sqrt{-g}} \partial_{\theta} \sqrt{-g} g^{\theta r} \partial_{r} \\
& +\frac{1}{\sqrt{-g}} \partial_{\theta} \sqrt{-g} g^{\theta \theta} \partial_{\theta}+\frac{1}{\sqrt{-g}} \partial_{\varphi} \sqrt{-g} g^{\varphi \varphi} \partial_{\varphi},
\end{aligned}
$$

or explicitly

$$
\begin{aligned}
\square \psi= & -L^{-1} \partial_{t}^{2} \psi+\frac{1}{\sqrt{-g}} \partial_{r} \sqrt{-g} L \partial_{r} \psi-\frac{1}{\sqrt{-g}} \partial_{\theta} \sqrt{-g} \frac{L^{1 / 2} b}{r f_{1}} \partial_{r} \psi-\frac{1}{\sqrt{-g}} \partial_{r} \sqrt{-g} \frac{L^{1 / 2} b}{r f_{1}} \partial_{\theta} \psi \\
& +\frac{1}{\sqrt{-g}} \partial_{\theta} \sqrt{-g}\left(r f_{1}\right)^{-2} \partial_{\theta} \psi+\frac{1}{\sqrt{-g}} \partial_{\varphi} \sqrt{-g}\left(r f_{2}\right)^{-2} \partial_{\varphi} \psi=0 .
\end{aligned}
$$

First, we study the equation $\square \psi=0$ perturbatively in $O(C) / K$ taking $\psi=\psi_{0}+\psi_{1}+\psi_{2}+\cdots$ and $\square=\square_{0}+\square_{1}+\square_{2}+\cdots$, where subscripts denote the orders in $O(C) / K$. We have

$$
\square \psi=\square_{0} \psi_{0}+\left(\square_{1} \psi_{0}+\square_{0} \psi_{1}\right)+\left(\square_{2} \psi_{0}+\square_{0} \psi_{2}+\square_{1} \psi_{1}\right)+\cdots .
$$


Expanding the terms in (3.2) in the series in $O(C) / K$, and using notations (2.22)(2.27), we have

$$
\begin{aligned}
& \frac{1}{\sqrt{-g}} \partial_{r} \sqrt{-g} L \partial_{r} \psi=\left[L \partial_{r}+L(2 / r)+\left(\partial_{r} L\right)+L\left(\partial_{r} g_{1}\right)+L\left(\left(\partial_{r} g_{2}-g_{1}\left(\partial_{r} g_{1}\right)\right)+\cdots\right] \partial_{r} \psi\right. \\
& \frac{1}{\sqrt{-g}} \partial_{\theta} \sqrt{-g} \frac{L^{1 / 2} b}{r f_{1}} \partial_{r} \psi=\left(\frac{L^{1 / 2}}{r}\right)\left[g_{1}^{r \theta} \partial_{\theta}+\left(\cot \theta g_{1}^{r \theta}+\left(\partial_{\theta} g_{1}^{r \theta}\right)\right)\right. \\
& \left.+\left(g_{2}^{r \theta} \partial_{\theta}+\cot \theta g_{2}^{r \theta}+\left(\partial_{\theta} g_{2}^{r \theta}\right)+\left(\partial_{\theta} g_{1}\right) g_{1}^{r \theta}\right)+\cdots\right] \partial_{r} \psi \\
& \frac{1}{\sqrt{-g}} \partial_{r} \sqrt{-g} \frac{L^{1 / 2} b}{r f_{1}} \partial_{\theta} \psi=\left(\frac{L^{1 / 2}}{r}\right)\left[g_{1}^{r \theta} \partial_{r}+(2 / r) g_{1}^{r \theta}+\left(\partial_{r} g_{1}^{r \theta}\right)\right. \\
& \left.+\left(g_{2}^{r \theta} \partial_{r}+(2 / r) g_{2}^{r \theta}+\left(\partial_{r} g_{2}^{r \theta}\right)+\left(\partial_{r} g_{1}\right) g_{1}^{r \theta}\right)+\cdots\right] \partial_{\theta} \psi
\end{aligned}
$$

Numerical calculations [20] of the Hawking radiation in different modes and theoretical considerations [21] have shown that the main part of the energy of radiation is contained in the $s$-wave. Because of that, first, in the main order, we consider the $s$ - wave eigenmode and, next, at the end of the section, comment on the modes with $l>0$.

In the zero order in $O(C) / K$, written in tortoise variable $r_{*}=r+2 M \ln (r / 2 M-1)$, the operator $\square_{0} \psi$ is

$$
\square_{0} \psi=(r L)^{-1}\left[-\partial_{t}^{2}+\partial_{r_{*}}^{2}+L\left(\frac{2 M}{r^{3}}-\frac{\hat{K}^{2}(\theta, \varphi)}{r^{2}}\right)\right] r \psi,
$$

where $\hat{K}^{2}$ is the angular momentum operator. In calculation of the Hawking radiation it is important to determine the behavior of the eigenmodes in the vicinity of the horizon [13, $15,21]$. We look for solution of the equation for the eigenmodes in the region $r-2 M \ll M$ and set $r=2 M$ in the functions with regular behavior at $r=2 M$.

When acting on $\psi_{0}$ the derivatives $\partial_{r}$ produce the terms with powers of $L^{-1}$ which grow up as $r \rightarrow 2 M$. In the leading order in $L^{-1}$ we have

$$
\partial_{r} \frac{e^{i \omega r_{*}}}{r} \simeq \frac{e^{i \omega r_{*}}}{r} \frac{i \omega}{L}, \quad \partial_{r}^{2} \frac{e^{i \omega r_{*}}}{r} \simeq-\left(\frac{\omega^{2}}{L^{2}}+\frac{2 i M \omega}{L^{2} r^{2}}\right) \frac{e^{i \omega r_{*}}}{r}
$$

Acting on $O\left(C^{n}\right) / K^{n}$ derivatives $\partial_{r}$ produce the factor $L^{-1 / 2}$

$$
\partial_{r} \frac{O\left(C^{n}\right)}{K^{n}}=-n \frac{O\left(C^{n}\right)}{K^{n} r L^{1 / 2}}
$$

In the region $r \sim 2 M$, neglecting in the operator $\square_{0}$ the small term $L 2 M / r^{3}$, solution of the eq. (3.7) for the $s$-mode is obtained as

$$
\psi_{0}=\frac{e^{i \omega\left(t \pm r_{*}\right)}}{\sqrt{4 \pi} r}
$$


Let us consider the equation (3.3) in the first order $\square_{1} \psi_{0}+\square_{0} \psi_{1}=0$. Using (3.4), (3.5) and (2.22), (2.24), we obtain (3.25) the terms without the derivatives $\partial_{\theta} \psi_{0}$ and $\partial_{\varphi} \psi_{0}$ in the operator $\square_{1} \psi_{0}$ as

$$
\begin{aligned}
{\left[L\left(\partial_{r} g_{1}\right)\right.} & +\left(-\frac{L^{1 / 2}}{r}\right)\left(\cot \theta g_{1}^{r \theta}+\left(\partial_{\theta} g_{1}^{r \theta}\right)\right] \partial_{r} \psi_{0} \\
& =\left[L \partial_{r}\left(-b^{\prime}-b \cot \theta\right)-\left(\frac{L^{1 / 2}}{r}\right)\left(b^{\prime}+b \cot \theta\right)\right] \partial_{r} \psi_{0}=0
\end{aligned}
$$

where we used the formula (3.9) to obtain $L \partial_{r} b=L \partial_{K}(2 C / K) \partial_{r} K=-b L^{1 / 2} / r$. The remaining terms in the operator $\square_{1} \psi_{0}$ contain derivatives $\partial_{\theta}$ and $\partial_{\varphi}$ acting on $\psi_{0}$ and vanish. The equation $\square_{1} \psi_{0}+\square_{0} \psi_{1}=0$ reduces to $\square_{0} \psi_{1}=0$ and yields $\psi_{1}=\psi_{0}$.

In the second order in $O(C) / K$ the equation (3.3) is $\square_{2} \psi_{0}+\square_{1} \psi_{1}+\square_{0} \psi_{2}=0$ which reduces to $\square_{2} \psi_{0}+\square_{0} \psi_{2}=0$. The terms without derivatives over angular variables acting on $\psi_{0}$ are

$$
\square_{2} \psi_{0}=\left[L \left(\left(\partial_{r} g_{2}-g_{1}\left(\partial_{r} g_{1}\right)\right)+\left(-\frac{L^{1 / 2}}{r}\right)\left(\cot \theta g_{2}^{r \theta}+\left(\partial_{\theta} g_{1}\right) g_{1}^{r \theta}+\left(\partial_{\theta} g_{2}^{r \theta}\right)\right] \partial_{r} \psi_{0}\right.\right.
$$

Both terms in the square brackets are of order $L^{1 / 2}$. In the leading order in $L^{-1}$ we have

$$
\square_{2} \psi_{0}=\frac{ \pm i \omega}{L^{1 / 2}} \frac{F_{(2)}(\theta, r)}{K^{2}} \psi_{0},
$$

(3.25) where $F_{(2)}=O\left(C^{2}\right)$, and the explicit form of $F_{(2)}$ is irrelevant for us. The equation $\square_{0} \psi_{2}=-\square_{2} \psi_{0}$ has the following structure

$$
\frac{1}{r L}\left[-\partial_{t}^{2}+\partial_{r_{*}}^{2}+L\left(\frac{2 M}{r^{3}}-\frac{\hat{K}^{2}(\theta, \varphi)}{r^{2}}\right)\right] r \psi_{2}=\frac{\mp i \omega}{L^{1 / 2}} \frac{F_{(2)}}{K^{2}} \frac{e^{i \omega\left(t \pm r_{*}\right)}}{r}
$$

Looking for a solution in the form $r \psi_{2}=f e^{i \omega\left(t \pm r_{*}\right)}$, in the leading order in $L^{-1}$ we obtain

$$
\partial_{r_{*}}^{2} f \pm 2 i \omega \partial_{r_{*}} f= \pm i \omega L^{1 / 2} \frac{F_{(2)}}{K^{2}}
$$

In the region $r \simeq 2 M$ approximately $r_{*}=2 M \ln L$. Solving the equation (3.15), we have

$$
\psi_{2}= \pm i \omega \frac{F_{(2)}}{K^{2}} \frac{L^{1 / 2}(4 M)^{2}}{1 \pm 8 i \omega M} \psi_{0}
$$

In the higher orders we proceed by induction. The equation for $\psi_{n}$ is

$$
\square_{0} \psi_{n}+\sum_{k+r=n, k, r \neq 0} \square_{k} \psi_{r}+\square_{n} \psi_{0}=0 .
$$

Let $\psi_{r}$ have the following structure

$$
\psi_{r}=L^{1 / 2} F\left(O\left(C^{k} / K^{k}\right), \theta, r\right) \psi_{0}, \quad k \lesssim r,
$$


The operators $\square_{k}, k>2$ can be presented in a form

$$
L\left(\partial_{r} F_{1}\right) \partial_{r}+\partial_{\theta} L^{1 / 2} F_{2} \partial_{r}+\partial_{r} L^{1 / 2} F_{3} \partial_{\theta},
$$

where $F_{i}=F_{i}\left(O\left(C^{l} / K^{l}\right), \theta, r\right) \sim O\left(L^{0}\right)$. When acting on the functions (3.18) each term in (3.17) yields an expression of the form (3.18) without the prefactor $L^{1 / 2}$ i.e. the result is of order $O\left(L^{0}\right)$. The operator $\square_{n}$ is of the same structure as (3.19), but when acting on the function $\psi_{0}$, it produces an expression of order $L^{-1 / 2}$. Eq. (3.17) reduces to $\square_{0} \psi_{n}+\square_{n} \psi_{0}=$ 0 which is of the functional form similar to (3.14) and yields solution

$$
\psi_{n} \sim L^{1 / 2} O\left(C^{n} / K^{n}\right) \psi_{0} .
$$

The origin of this result can be traced back to the form of d'Alambertian (3.2) in which the $(t, r)$ part is the same as in the Schwarzschild metric and the additional $(r, \theta)$ terms contain the factor $L^{1 / 2}$.

Let us consider solutions with higher harmonics, $l>0$. Approximate solution of the equation $\square_{0} \psi_{0 l}=0$ in the main order in $L^{-1}$ with the $l$-th harmonic is

$$
\psi_{l, 0}=\frac{e^{i \omega\left(t \pm r_{*}\right)}}{\sqrt{4 \pi} r} P_{l}(\cos \theta) .
$$

Now the terms containing $\partial_{\theta} \psi_{l, k}$ are nonbibitem-zero. In the next order, in the operator $\square_{1} \psi_{l-1,0}$, there appears the new term

$$
\left.\frac{1}{\sqrt{g}} \partial_{r} \sqrt{g}\right|_{(0)} g_{1}^{\theta r} \partial_{\theta} \psi_{l-1,0}=\frac{1}{r^{2}} \partial_{r} r^{2} \frac{L^{1 / 2}}{r} \hat{g}_{1}^{\theta r} \partial_{\theta} \psi_{l-1,0} .
$$

Because $\partial_{\theta}$ does not change the order in $L$, this term is of order $L^{1 / 2}$ and can be neglected. In the next orders we find similar situation. New terms with the derivative $\partial_{\theta}$ do not increase powers of $L^{-1}$. As a result, we obtain solution of the form

$$
\psi_{l} \sim L^{1 / 2} \frac{F_{(n)}}{K^{n}} \psi_{0}
$$

Above we assumed that $C(\theta)=\sum P_{n}\left(\cos ^{2} \theta\right)$. Because the action of $\partial_{\theta}$ on $P_{n}\left(\cos ^{2} \theta\right)$ produces factor $\mathrm{n}$, in the higher orders in $O(C) / K$ in the terms $\square_{k} \psi_{n}$, in principle, accumulate powers of $n$. Trying to sum all orders, we encounter the problem of convergence. To avoid this problem, we consider non-perturbative solution of the equation (3.3).

We present $\square$ as the sum $\square_{0}+\hat{\square}$, where $\square_{0}$ is the zero-order part of d'Alambertian and $\hat{\square}$ written in notations $(2.22)-(2.27)$ is

$$
\begin{aligned}
\hat{\square}= & \frac{1}{\sqrt{-\hat{g}}} \partial_{r} \sqrt{-\hat{g}} L \partial_{r}-\frac{1}{\sqrt{-g}} \partial_{\theta} \sqrt{-g} \frac{L^{1 / 2} b}{r f_{1}} \partial_{r}-\frac{1}{\sqrt{-g}} \partial_{r} \sqrt{-g} \frac{L^{1 / 2} b}{r f_{1}} \partial_{\theta} \\
& +r^{-2}\left(f_{1}^{2}-1\right)\left(\partial_{\theta}^{2}+\cot \theta \partial_{\theta}\right)+\frac{1}{\sqrt{-\hat{g}}} \partial_{\theta} \sqrt{-\hat{g}}\left(r f_{1}\right)^{-2} \partial_{\theta}+r^{-2}\left(f_{2}^{-2}-1\right) \partial_{\varphi}^{2} .
\end{aligned}
$$

We look for a solution $\psi$ in the form $\psi_{0}+\hat{\psi}$, where $\psi_{0}$ is the zero-order $s$-mode. Equation $\square \psi=0$ takes the form

$$
\square_{0} \hat{\psi}+\hat{\square} \psi_{0}+\hat{\square} \hat{\psi}=0
$$


We take an ansatz for $\hat{\psi}$ in a form $L^{1 / 2} \varphi \psi_{0}$, where $\varphi$ is sufficiently smooth function and $L^{1 / 2} \partial_{r} \varphi$ is finite in the limit $L=0$. We have

$$
\partial_{r} \hat{\psi}=\partial_{r}\left(L^{1 / 2} \varphi \psi_{0}\right)=\left(\frac{M}{r^{2}} \pm i \omega\right) L^{-1 / 2} \varphi \psi_{0}+L^{1 / 2}\left(\partial_{r} \varphi\right) \psi_{0}
$$

Let us consider $\hat{\square} \psi_{0}$. The terms with derivatives $\partial_{\theta}$ and $\partial_{\varphi}$ acting on $\psi_{0}$ yield zero. We have

$$
\hat{\square} \psi_{0}=\frac{1}{\sqrt{-\hat{g}}} L\left(\partial_{r} \sqrt{-\hat{g}}\right) \partial_{r} \psi_{0}+\frac{1}{\sqrt{-g}} \partial_{\theta} \sqrt{-g} \frac{L^{1 / 2} b}{r f_{1}} \partial_{r} \psi_{0}= \pm i \omega L^{-1 / 2}\left(F_{1}+F_{2}\right) \psi_{0},
$$

where

$$
\frac{1}{\sqrt{-\hat{g}}} L\left(\partial_{r} \sqrt{-\hat{g}}\right) \partial_{r} \psi_{0}= \pm i \omega L^{-1 / 2} F_{1} \psi_{0}
$$

and

$$
\frac{1}{\sqrt{-g}} \partial_{\theta} \sqrt{-g} \frac{L^{1 / 2} b}{r f_{1}} \partial_{r} \psi_{0}= \pm i \omega L^{-1 / 2} F_{2} \psi_{0}
$$

Next, let us consider the term $\square \hat{\psi}$. The $(r r)$ term is

$$
L \frac{\left(\partial_{r} \sqrt{-\hat{g}}\right)}{\sqrt{-\hat{g}}} \partial_{r} \hat{\psi}=F_{1}\left(\frac{M}{r^{2}} \pm i \omega\right) L^{-1 / 2} \varphi \psi_{0}+F_{1} L^{1 / 2}\left(\partial_{r} \varphi\right) \psi_{0} .
$$

The $(\theta r)$ term is

$$
\begin{aligned}
\frac{1}{\sqrt{-g}} \partial_{\theta} \sqrt{-g} \frac{L^{1 / 2} b}{r f_{1}} \partial_{r} \hat{\psi} & =L^{1 / 2}\left[\left(\partial_{\theta} F_{2}\right) \partial_{r} \hat{\psi}+F_{2} \partial_{r} \partial_{\theta} \hat{\psi}\right] \\
& =L^{-1 / 2}\left(\frac{M}{r^{2}} \pm i \omega\right)\left[\left(\partial_{\theta} F_{2}\right) \varphi+F_{2}\left(\partial_{\theta} \varphi\right)\right] \psi_{0}
\end{aligned}
$$

and the $(r \theta)$ term is

$$
\frac{1}{\sqrt{-g}} \partial_{r} \sqrt{-g} \frac{L^{1 / 2} b}{r f_{1}} \partial_{\theta} \hat{\psi}=L^{-1 / 2} F_{3} \partial_{\theta} \hat{\psi}+L^{1 / 2} F_{2} \partial_{r} \partial_{\theta} \hat{\psi}
$$

Collecting the leading in $L^{-1}$ terms, we obtain eq. (3.21) as

$$
\square_{0} \hat{\psi}+L^{-1 / 2}\left(F_{1}+F_{2}\right) i \omega \psi_{0}+L^{-1 / 2}\left(\Psi_{1} \varphi \psi_{0}+\Psi_{2} \partial_{\theta} \varphi \psi_{0}\right)=0 .
$$

eq. (3.25) has the same functional form as eq. (3.14), and we conclude that the ansatz $\hat{\psi}=L^{1 / 2} \varphi \psi_{0}$ yields a solution of (3.25).

\section{Isotropic geodesics}

To calculate the number of particles emitted to the future null infinity $I_{+}$one propagates the wave-packet backwards from $I_{+}$along a null geodesic $[13,17]$. In this section we consider a class of the isotropic geodesics in the metric (2.19) depending on $r$ and $\theta$ and show that in the vicinity of horizon they differ from geodesics in the Schwarzschild background by the 
terms of order $L^{1 / 2}=(1-2 M / r)^{1 / 2}$. Following the treatment of [22] we start from the Lagrangian corresponding to the metric (2.19) which we present as

$$
2 \mathcal{L}=-L \dot{t}^{2}+\frac{\dot{r}^{2}}{L} \hat{g}_{r r}+2 \frac{r \dot{r} \dot{\theta}}{L^{1 / 2}} \hat{g}_{r \theta}+r^{2}\left[\dot{\theta}^{2} \hat{g}_{\theta \theta}+\dot{\varphi}^{2} \sin ^{2} \theta \hat{g}_{\varphi \varphi}\right] .
$$

Derivatives are taken with respect to an affine parameter on geodesic. The Lagrange equations following from the Lagrangian yield the geodesic equations for $r(\tau), t(\tau)$ and $\theta(\tau), \varphi(\tau)$. The equations for $r(\tau)$ and $\theta(\tau)$ are

$$
\begin{aligned}
\frac{\ddot{r}}{L} \hat{g}_{r r}- & \frac{\dot{r}^{2}}{r^{2} L^{2}} \hat{g}_{r r}+\frac{E^{2}}{r^{2} L^{2}}+\frac{\dot{r}^{2}}{2 L} \partial_{r} \hat{g}_{r r}+\frac{\dot{r} \dot{\theta}}{L} \partial_{\theta} \hat{g}_{r r} \\
& +\frac{\ddot{\theta} r}{L^{1 / 2}} \hat{g}_{r \theta}+\dot{\theta}^{2}\left(\frac{r}{L^{1 / 2}} \partial_{\theta} \hat{g}_{r \theta}-r \hat{g}_{\theta \theta}-\frac{r^{2}}{2} \partial_{r} \hat{g}_{\theta \theta}\right)-\dot{\varphi}^{2}\left(r \hat{g}_{\varphi \varphi}+\frac{r^{2}}{2} \partial_{r} \hat{g}_{\varphi \varphi}\right)=0 \\
\ddot{\theta} r^{2} \hat{g}_{\theta \theta}+ & 2 r \dot{\theta} \dot{r} \hat{g}_{\theta \theta}+r^{2} \dot{\theta} \dot{r} \partial_{r} \hat{g}_{\theta \theta}+\frac{1}{2} r^{2} \dot{\theta}^{2} \partial_{\theta} \hat{g}_{\theta \theta}+\frac{\ddot{r} r+\dot{r}^{2}}{L^{1 / 2}} \hat{g}_{r \theta}-\frac{\dot{r}^{2}}{r L^{3 / 2}} \hat{g}_{r \theta} \\
& +\frac{\dot{r}^{2} r}{L^{1 / 2}} \partial_{r} \hat{g}_{r \theta}-\frac{\dot{r}^{2}}{2 L} \partial_{\theta} \hat{g}_{r r}-\dot{\varphi}^{2} r^{2}\left(\sin \theta \cos \theta \hat{g}_{\varphi \varphi}+\sin ^{2} \theta \partial_{\theta} \hat{g}_{\varphi \varphi}\right)=0
\end{aligned}
$$

Here $\partial_{r} \hat{g}_{i j}=K \partial_{K} \hat{g}_{i j} / L^{1 / 2} r$. Because $t$ and $\varphi$ are cyclic variables, we solved the corresponding equations and set in the Lagrange equations $\dot{t}=E / L$ and $r^{2} \dot{\varphi} \sin ^{2} \theta=B=$ const. We consider the geodesics with $B=0$.

For the isotropic geodesics equation $\mathcal{L}=0$ is the first integral of the system of geodesic equations

$$
-\frac{E^{2}}{2 L}+\frac{\dot{r}^{2}}{2 L} \hat{g}_{r r}+\frac{r \dot{r} \dot{\theta}}{L^{1 / 2}} \hat{g}_{r \theta}+\frac{r^{2}}{2} \dot{\theta}^{2} \hat{g}_{\theta \theta}=0 .
$$

In its general form the system of equations is intractable. To proceed, we consider the near-horizon regon $r \rightarrow 2 M$. In this limit $L \rightarrow 0$. Examining the equations (4.2), (4.3) and (4.4) we see that in the near-horizon limit we can look for a solution in the form

$$
\begin{aligned}
& \dot{r}=C+C_{1} L^{1 / 2}+\cdots, \\
& \dot{\theta}=\frac{A}{L^{1 / 2}}+A_{1}+\cdots, \quad \ddot{\theta}=-\frac{A \dot{r}}{r^{2} L^{3 / 2}}+\cdots .
\end{aligned}
$$

With the ansatz (4.5) the leading in $L^{-1}$ terms in (4.2) and (4.3) are

$$
\begin{aligned}
\frac{E^{2}}{r^{2} L^{2}}-\frac{\dot{r}^{2}}{r^{2} L^{2}} \hat{g}_{r r}+\frac{\ddot{\theta} r}{L^{1 / 2}} \hat{g}_{r \theta}=0, \\
\ddot{\theta} r^{2} \hat{g}_{\theta \theta}-\frac{\dot{r}^{2}}{r L^{1 / 2}} \hat{g}_{r \theta}=0 .
\end{aligned}
$$

Substituting the ansatz (4.5), we obtain

$$
\begin{aligned}
L^{-2}\left[E^{2}-C^{2} \bar{g}_{r r}-r A C \bar{g}_{r \theta}\right] & =0, \\
L^{-3 / 2}\left[C \bar{g}_{r \theta}+r A \bar{g}_{\theta \theta}\right] & =0 .
\end{aligned}
$$

and (4.4) is

$$
L^{-1}\left[C^{2} \bar{g}_{r r}-E^{2}+2 r C A \bar{g}_{r \theta}+r^{2} A^{2} \bar{g}_{\theta \theta}\right]=0
$$


Here we have introduced $\bar{g}_{i j}=\left.\hat{g}_{i j}\right|_{r=2 M}$. Note that $\hat{g}_{i j}$ are the components of the metric without the factors $L$ (see (2.24). Using the relation

$$
\hat{g}_{r r} \hat{g}_{\theta \theta}-\hat{g}_{r \theta}^{2}=\hat{g}_{\theta \theta},
$$

from the eqs. (4.6) and (4.7) we obtain

$$
\begin{aligned}
C^{2} & =E^{2}, \\
A & \simeq-\frac{C \bar{g}_{r \theta}}{2 M \bar{g}_{\theta \theta}} .
\end{aligned}
$$

Because of the relation (4.11), eq. (4.10) turns to identity.

In the limit $b=0$ the Lagrangian reduces to that of the Schwarzschild metric. In the spherically-symmetric metrics trajectories of the geodesics are located in a plane going through the symmetry center. Position of the plane depends on the initial conditions. $\theta$ and $\varphi$ are coordinates in a coordinate system with the origin located at the symmetry center. Position of the plane depends on the initial conditions. Solution of the radial geodesic equations with the initial conditions $\theta\left(\tau_{0}\right)=\pi / 2, \dot{\theta}\left(\tau_{0}\right)=0, \varphi\left(\tau_{0}=\dot{\varphi}\left(\tau_{0}\right)=0\right.$ is

$$
\begin{aligned}
\dot{t}^{2} & =E^{2}=\dot{r}^{2} \\
\theta(\tau) & =\pi / 2, \\
\varphi(\tau) & =0 .
\end{aligned}
$$

Comparing solution (4.12), (4.13) with that in the Schwarzschild metric and adjusting the integration constants, we obtain

$$
\begin{aligned}
r & =2 M+E \tau, \\
t & =t_{0}+E \tau, \\
\theta & \simeq \frac{\pi}{2}-\frac{\bar{g}_{r \theta}}{2 \bar{g}_{\theta \theta}} \sqrt{\frac{\tau E}{2 M}}=\frac{\pi}{2}-\frac{\bar{g}_{r \theta}}{2 \bar{g}_{\theta \theta}} L^{1 / 2} .
\end{aligned}
$$

In the vicinity of horizon solution (4.15) differ from (4.14) by the terms of order $L^{1 / 2}$.

\section{Conclusions}

In this paper we discussed several aspects of calculation of Hawking radiation of black holes with supertranslation field. In the standard calculation $[13,14]$ one considers propagation of a massles scalar field in the metric of black hole. The field satisfies the wave equation with the initial data on a hypersurface usually chosen as the past null infinity $I^{-}$. The field is expanded in the orthonormal set of positive-frequency modes $\left\{u_{\omega}^{(-)}\right\}$at $I^{-}: u_{\omega}^{(-)} \sim e^{-i \omega v}$ Alternatively, as a hypersurface of initial data can be chosen the union of the future null infinity $I^{+}$and horizon. On $I^{+}$the field is expanded in the orthonormal set of modes $\left\{u_{\omega}^{(+)}\right\}$ which are positive-frequency on $I^{+}: u_{\omega}^{(+)} \sim e^{-i \omega u}$ and a set of modes on $H$ which have no outgoing components. The modes $\left\{u_{\omega}^{(+)}\right\}$can be expanded in terms of the modes $\left\{u_{\omega}^{(-)}\right\}$

$$
u_{\omega}^{(+)}=\int d \omega^{\prime}\left(\alpha_{\omega \omega^{\prime}} u_{\omega^{\prime}}^{(-)}+\beta_{\omega \omega^{\prime}} u_{\omega^{\prime}}^{(-) *}\right) .
$$


The particle content of the outgoing mode expressed through the particle basis defined with respect to the vacuum at $I^{-}$is

$$
<i n\left|N_{\omega}\right| i n>=\int d \omega^{\prime} \beta_{\omega_{1} \omega^{\prime}} \beta_{\omega_{2} \omega^{\prime}}^{*}
$$

The main contribution to $\beta_{\omega \omega^{\prime}}$ is produced by the modes propagating from $I^{-}$and passing through the collapsing body just before the horizon was formed. In actual calculation the outgoing modes are propagated backwards and expanded in $u_{\omega}^{(-)}$near horizon. The problem reduces to finding solution of the wave equation in the near-horizon region (requirement of horizon formation can be weaken to a variety of more general cases [16, 17, 21]).

We study solutions of the wave equation in the near-horizon limit in the case of the Schwarzschild metric with the supertranslation field $C(\theta)$. We find that in any order of perturbation theory in $O(C) / M$, in the vicinity of horizon, solutions for the eigenmodes in the metric with supertranslation field differ from solutions in the pure Schwarzschild background by the terms of order $L^{1 / 2} O\left(C^{n} / M^{n}\right)$ where $L=(1-2 M / r)$. The same result is valid for the non-perturbative solution, in which case the difference between solutions for the eigenmodes in the pure Schwarzschild background, $\psi_{0}$, and in the Schwarzschild background with supertranslation field is $L^{1 / 2} \varphi(\theta, r) \psi_{0}$, where $L^{1 / 2} \partial_{r} \varphi$ is finite in the limit $L=0$.

In background of any metric, the wave packets of the massles field propagate following the isotropic geodesics in this metric. We study the form of isotropic geodesics in the Schwarzschild metric with supertranslation field. Because of $\theta$-dependence of the supertranslation field, the form of geodesics is considerably more complex than in the spherically-symmetric case. However, we show that in the near-horizon region, as $r \rightarrow 2 M$ the radial geodesics differ from the geodesics in the pure Schwarzschild case by terms of order $(1-2 M / r)^{1 / 2}$.

\section{Acknowledgments}

I am grateful to L. Slad and M. Smolyakov for useful discussions. This work was partially supported by the Ministry of Science and Education of Russian Federation under the project 01201255504 .

Open Access. This article is distributed under the terms of the Creative Commons Attribution License (CC-BY 4.0), which permits any use, distribution and reproduction in any medium, provided the original author(s) and source are credited.

\section{References}

[1] H. Bondi, M.G.J. van der Burg and A.W.K. Metzner, Gravitational waves in general relativity. 7. Waves from axisymmetric isolated systems, Proc. Roy. Soc. Lond. A 269 (1962) 21 [INSPIRE].

[2] R.K. Sachs, Gravitational waves in general relativity. 8. Waves in asymptotically flat space-times, Proc. Roy. Soc. Lond. A 270 (1962) 103 [INSPIRE]. 
[3] G. Barnich and G. Compere, Classical central extension for asymptotic symmetries at null infinity in three spacetime dimensions, Class. Quant. Grav. 24 (2007) F15 [gr-qc/0610130] [INSPIRE].

[4] G. Barnich and C. Troessaert, Aspects of the BMS/CFT correspondence, JHEP 05 (2010) 062 [arXiv: 1001.1541] [INSPIRE].

[5] G. Barnich and C. Troessaert, Symmetries of asymptotically flat 4 dimensional spacetimes at null infinity revisited, Phys. Rev. Lett. 105 (2010) 111103 [arXiv:0909.2617] [INSPIRE].

[6] A. Strominger, On BMS invariance of gravitational scattering, JHEP 07 (2014) 152 [arXiv: 1312.2229] [INSPIRE].

[7] G. Compère and J. Long, Classical static final state of collapse with supertranslation memory, Class. Quant. Grav. 33 (2016) 195001 [arXiv:1602.05197] [INSPIRE].

[8] A. Strominger and A. Zhiboedov, Gravitational memory, BMS supertranslations and soft theorems, JHEP 01 (2016) 086 [arXiv:1411.5745] [INSPIRE].

[9] S. Weinberg, Infrared photons and gravitons, Phys. Rev. 140 (1965) B516.

[10] S.W. Hawking, M.J. Perry and A. Strominger, Soft hair on black holes, Phys. Rev. Lett. 116 (2016) 231301 [arXiv:1601.00921] [InSPIRE].

[11] G. Compère and J. Long, Vacua of the gravitational field, JHEP 07 (2016) 137 [arXiv: 1601.04958] [INSPIRE].

[12] A. Strominger, Black hole information revisited, arXiv:1706.07143 [INSPIRE].

[13] S.W. Hawking, Particle Creation by Black Holes, Commun. Math. Phys. 43 (1975) 199 [Erratum ibid. 46 (1976) 206] [INSPIRE].

[14] N.D. Birrell and P.C.W. Davies, Quantum Fields in Curved Space, Cambridge University Press, Cambridge U.K. (1982).

[15] R. Brout, S. Massar, R. Parentani and P. Spindel, A primer for black hole quantum physics, Phys. Rept. 260 (1995) 329 [arXiv:0710.4345] [InSPIRE].

[16] L.C. Barbado, C. Barcelo and L.J. Garay, Hawking radiation as perceived by different observers, Class. Quant. Grav. 28 (2011) 125021 [arXiv:1101.4382] [INSPIRE].

[17] C. Barcelo, S. Liberati, S. Sonego and M. Visser, Hawking-like radiation from evolving black holes and compact horizonless objects, JHEP 02 (2011) 003 [arXiv: 1011.5911] [INSPIRE].

[18] S.J. Fletcher and A.W.C. Lun, The Kerr spacetime in generalized Bondi-Sachs coordinates, Class. Quant. Grav. 20 (2003) 4153.

[19] G. Barnich and C. Troessaert, Comments on holographic current algebras and asymptotically flat four dimensional spacetimes at null infinity, JHEP 11 (2013) 003 [arXiv: 1309.0794] [INSPIRE].

[20] N.G. Sanchez, Absorption and emission spectra of a Schwarzschild black hole, Phys. Rev. D 18 (1978) 1030 [INSPIRE].

[21] M. Visser, Essential and inessential features of Hawking radiation, Int. J. Mod. Phys. D 12 (2003) 649 [hep-th/0106111] [INSPIRE].

[22] S. Chandrasekhar, The mathematical theory of black holes, Oxford University Press, Oxford U.K. (1983). 